\title{
General Structure of Nonlinear Evolution Equations in $1+2$ Dimensions Integrable by The Two-Dimensional Gelfand-Dickey-Zakharov-Shabat Spectral Problem and their Transformation Properties
}

\author{
B. G. Konopelchenko
}

Institute of Nuclear Physics, Novosibirsk-90, 630090, USSR

\begin{abstract}
The general form of nonlinear evolution equations connected with the matrix two-dimensional Gelfand-Dickey-Zakharov-Shabat spectral problem is found. The infinite-dimensional abelian group of general Bäcklund transformations and infinite-dimensional abelian symmetry group for these equations are constructed.
\end{abstract}

\section{Introduction}

One of the main problems of the inverse scattering transform (IST) method is the description of equations integrable by this method (see e.g. [1,2]). All the equations to which the IST method is applicable form the classes of the equations integrable by the same spectral problem. A very convenient and simple description of the partial differential equations integrable by the spectral problem

$$
\frac{\partial \Psi}{\partial x}=\lambda A \Psi+P(x, t) \Psi
$$

of the second matrix order has been given by Ablowitz, Kaup, Newell and Segur in [3]. Then this approach (AKNS approach) has been generalised to the problem (1.0) of an arbitrary order [4-10], to some other spectral problems $[11,12]$ and, in particular, to the one-dimensional Gelfand-Dickey spectral problem [13].

Recently the two-dimensional generalisation of the AKNS technique has been developed [14]. Namely, the two-dimensional arbitrary order spectral problem $\partial \Psi / \partial x+A(\partial \Psi / \partial y)+P(x, y, t) \Psi=0$, where $A$ is any diagonalisable constant matrix was considered: the general form of the nonlinear equations integrable by this problem and their Bäcklund transformations were found [14].

In the present paper we consider the two-dimensional matrix Gelfand-Dickey- 
Zakharov-Shabat problem

$$
\frac{\partial^{N} \chi}{\partial x^{N}}+V_{N-2}(x, y, t) \frac{\partial^{N-2} \chi}{\partial x^{N-2}}+\cdots+V_{1}(x, y, t) \frac{\partial \chi}{\partial x}+V_{0}(x, y, t) \chi+\frac{\partial \chi}{\partial y}=0,
$$

where $N$ is any integer, the coefficients $V_{0}(x, y, t), \quad V_{1}(x, y, t), \cdots$, $V_{N-2}(x, y, t)$ are matrices of an arbitrary order $M$ which depend on the two coordinates $x, y$ and time $t$, and $V_{k}(x, y, t) \underset{\sqrt{x^{2}+y^{2}} \rightarrow \infty}{\longrightarrow} 0(k=0,1, \ldots, N-2)$. The applicability of the IST method to the problem $(1.1)$ was discussed in $[15,16]$. In the case $N=2$ the problem (1.1) was used for the integration of the KadomtsevPetviashvili equation $[15,17,18]$.

We find the general form of nonlinear evolution equations in $1+2$ dimensions $(t, x, y)$ integrable by the problem (1.1). We construct the infinite-dimensional abelian group of general Bäcklund transformations and the infinite-dimensional abelian symmetry group for these equations. As an example we consider the case $N=2$. In this case we also obtain the nonlinear superposition formulas for the simplest Bäcklund transformation.

The paper is organised as follows. In the second section we rewrite the problem (1.1) in a matrix form, then we consider the direct scattering problem and obtain some important relations. In Sect. 3 we calculate the recursion operators which play a main role in our constructions. The general form of the integrable equations and Bäcklund transformations are found in Sect. 4. Group-theoretical properties of the integrable equations are discussed briefly in Sect. 5. In Sect. 6 the case $N=2$ is considered: the infinite family of the equations, the simplest of which is the Kadomtsev-Petviashvili equation, their Bäcklund transformations and nonlinear superposition formulas are described.

\section{Direct Scattering Problem and Some Important Relations}

Let us note first of all that the problem (1.1) is equivalent to a matrix problem of order $N M$

$$
\frac{\partial \hat{\Psi}}{\partial x}+A \frac{\partial \hat{\Psi}}{\partial y}+P(x, y, t) \hat{\Psi}=0
$$

where

$$
A=\left(\begin{array}{cccc}
0 & 0 & \ldots & 0 \\
\ldots & \ldots & \ldots & \ldots \\
0 & 0 & \ldots & 0 \\
I_{M} & 0 & \ldots & 0
\end{array}\right), P=\left(\begin{array}{ccccc}
0 & -I_{M} & 0 & \ldots & 0 \\
0 & 0 & -I_{M} & \ldots & 0 \\
\ldots & \ldots & \ldots & \ldots & \ldots \\
0 & 0 & \ldots & 0 & -I_{M} \\
V_{0} & V_{1} & \ldots & V_{N-2} & 0
\end{array}\right)
$$

and $I_{M}$ is an $M \times M$ unity matrix. The adjoint spectral problem is

$$
\frac{\partial \breve{\Psi}}{\partial x}+\frac{\partial \check{\Psi}}{\partial y} A-\check{\Psi} P(x, y, t)=0 \text {. }
$$


The matrix problems (2.1) and (2.3) are more convenient for our purpose than problem (1.1) and the problem adjoint to (1.1).

Let us consider first the direct scattering problem for (2.1) and (2.3). We follow $[16,14]$. We assume that $V_{k}(x, y, t) \rightarrow 0$ at $\sqrt{x^{2}+y^{2}} \rightarrow \infty$ sufficiently rapidly that all quantities and integrals exist and that $\int_{-\infty}^{+\infty} d y \frac{\partial}{\partial y}(\cdots)=0$.

We introduce the matrices-solutions $\hat{F}_{\lambda}^{+}(x, y, t)$ and $\hat{F}_{\lambda}^{-}(x, y, t)$ of the problem (2.1) given by their asymptotic behaviour

$$
\begin{gathered}
\hat{F}_{\lambda}^{+}(x, y, t) \underset{x \rightarrow+\infty}{\longrightarrow}(2 \pi i)^{-1 / 2} D(\lambda) \exp \left\{-\lambda^{N} y+\bar{A} x\right\}, \\
\hat{F}_{\lambda}^{-}(x, y, t) \underset{x \rightarrow-\infty}{\longrightarrow}(2 \pi i)^{-1 / 2} D(\lambda) \exp \left\{-\lambda^{N} y+\bar{A} x\right\},
\end{gathered}
$$

where $\lambda$ is a complex number, $\bar{A}$ is a diagonal matrix:

$$
\bar{A}_{i k}=\lambda q^{i-1} \delta_{i k}\left(q=\exp \frac{2 \pi i}{N}, \delta_{i k}=\left\{\begin{array}{ll}
1, & i=k \\
0, & i \neq k
\end{array}\right)\right.
$$

and

$$
D_{i k}=\frac{1}{\sqrt{N}}\left(\lambda q^{(k-1)}\right) i^{-1} I_{M}(i, k=1, \ldots, N)^{1} .
$$

The quantities $\lambda q^{i-1}$ are eigenvalues of the matrix $\tilde{A}=\lambda^{N} A+P_{\infty}$, where $P_{\infty} \stackrel{\text { def }}{=}=-\lim _{\sqrt{x^{2}+y^{2} \rightarrow \infty}} P(x, y, t)$ and $\hat{A}=D \bar{A} D^{-1}$.

The scattering matrix $\hat{S}(\tilde{\lambda}, \lambda, t)$ for problem $(2.1)$ is defined as follows

$$
\hat{F}_{\lambda}^{+}(x, y, t)=\int_{-i \infty}^{+i \infty} d \tilde{\lambda} \hat{F}_{\tilde{\lambda}}^{-}(x, y, t) \hat{S}(\tilde{\lambda}, \lambda, t) .
$$

Correspondingly for the adjoint problem (2.3) we introduce matrices-solutions $\check{F}_{\lambda}^{+}(x, y, t)$ and $\check{F}_{\lambda}^{-}(x, y, t)$ for which:

$$
\check{F}_{\lambda}^{ \pm}(x, y, t) \underset{x \rightarrow \pm \infty}{\longrightarrow}(2 \pi i)^{-1 / 2} \exp \left\{\lambda^{N} y-\bar{A} x\right\} D^{-1}(\lambda)
$$

and the scattering matrix $\breve{S}(\widetilde{\lambda}, \lambda, t)$ :

$$
\check{F}_{\lambda}^{+}(x, y, t)=\int_{-i \infty}^{+i \infty} d \tilde{\lambda} \check{S}(\lambda, \tilde{\lambda}, t) \check{F}_{\tilde{\lambda}}^{-}(x, y, t) .
$$

It is not difficult to show using $(2.1)-(2.7)$ that the following relations hold:

$$
\int_{-\infty}^{+\infty} d y \check{F}_{\bar{\lambda}}^{ \pm}(x, y, t) \hat{F}_{\lambda}^{ \pm}(x, y, t)=\delta(\tilde{\lambda}-\lambda),
$$

1 Here and below latin indices take the values $1,2, \ldots, N$ (or $N-1$ ) and numerate the block elements of matrices of the order $N M$ which are themselves the matrices $M \times M$. Greek indices mark the usual matrix elements of $N M \times N M$ matrices and take the values $1,2, \ldots, N M$ 


$$
\begin{aligned}
\int_{-i \infty}^{+i \infty} d \lambda \hat{F}_{\lambda}^{ \pm}(x, y, t) \check{F}_{\lambda}^{ \pm}\left(x, y^{\prime}, t\right) & =\delta\left(y^{\prime}-y\right), \\
& \int_{-i \infty}^{+i \infty} d \mu \check{S}(\tilde{\lambda}, \mu, t) \hat{S}(\mu, \lambda, t)=\delta(\tilde{\lambda}-\lambda),
\end{aligned}
$$

where $\delta(\lambda)$ is the Dirac delta-function. Hence the scattering matrices can be represented as follows

$$
\begin{aligned}
& \hat{S}(\tilde{\lambda}, \lambda, t)=\int_{-\infty}^{+\infty} d y \check{F}_{\tilde{\lambda}}(x, y, t) \hat{F}_{\lambda}^{+}(x, y, t), \\
& \check{S}(\tilde{\lambda}, \lambda, t)=\int_{-\infty}^{+\infty} d y \check{F}_{\tilde{\lambda}}^{+}(x, y, t) \hat{F}_{\lambda}^{-}(x, y, t) .
\end{aligned}
$$

Now let $P$ and $P^{\prime}$ be two different matrices and $\hat{F}^{+}, \check{F}^{+}, \hat{F}^{+}, \hat{S}, \hat{S}^{\prime}$ be corresponding solutions and scattering matrices for the problems (2.1) and (2.3). One can prove (analogously to Ref. [14]) the following important relation

$$
\begin{aligned}
& \hat{S}^{\prime}(\tilde{\lambda}, \lambda, t)-\hat{S}(\tilde{\lambda}, \hat{\lambda}, t) \\
& \quad=-\int_{-i \infty}^{+i \infty} d \mu \hat{S}(\tilde{\lambda}, \mu, t) \int_{-\infty}^{+\infty} d x d y \check{F}_{\mu}^{+}(x, y, t)\left(P^{\prime}(x, y, t)-P(x, y, t)\right) \hat{F}_{\lambda}^{+{ }^{\prime}}(x, y, t) .
\end{aligned}
$$

The mapping $P(x, y, t) \rightarrow \hat{S}(\tilde{\lambda}, \lambda, t)$ given by the spectral problem $(2.1)$ establishes a correspondence between the transformations $B_{p}: P \rightarrow P^{\prime}$ of the manifold of the potentials $\{P(x, y, t)\}$ and the transformations $B_{S} . \hat{S} \rightarrow \hat{S}^{\prime}$ of the manifold of the scattering matrices $\{\hat{S}(\tilde{\lambda}, \lambda, t)\}$.

We consider only such transformations $B$ that

$$
\hat{S}(\tilde{\lambda}, \lambda, t) \stackrel{B}{\rightarrow} \hat{S}^{\prime}(\tilde{\lambda}, \lambda, t)=B^{-1}(\tilde{\lambda}, t) \hat{S}(\tilde{\lambda}, \lambda, t) C(\lambda, t)
$$

where $B(\tilde{\lambda}, t)$ and $C(\lambda, t)$ are arbitrary block diagonal matries, i.e. $B_{i k}=$ $B_{i}(\lambda, t) \delta_{i k} I_{M}, C_{i k}=C_{i}(\lambda, t) \delta_{i k} I_{M}$. This "restricted" class of the transformations, as we shall see, is wide enough.

Further, it is not difficult to show that the following identity holds

$$
\begin{gathered}
-\int_{-i \infty}^{+\infty} d \mu \check{S}(\tilde{\lambda}, \mu, t)(1-B(\mu, t)) \hat{S}^{\prime}(\mu, \lambda, t)+(1-B(\lambda, t)) \delta(\tilde{\lambda}-\lambda) \\
=\left.\int_{-\infty}^{+\infty} d y\left\{\check{F}_{\tilde{\lambda}}^{+}(x, y, t)\left(1-\tilde{B}\left(-\partial_{y}, t\right)\right) \hat{F}_{\lambda}^{++^{\prime}}(x, y, t)\right\}\right|_{x=-\infty} ^{x=+\infty} \\
=-\int_{-\infty}^{+\infty} d x d y \check{F}_{\tilde{\lambda}}^{+}(x, y, t)\left\{\tilde{P}(x, y, t)\left(1-\tilde{B}\left(-\partial_{y}, t\right)\right) \hat{F}_{\lambda}^{+{ }^{\prime}}(x, y, t)\right. \\
\left.-\left(1-\tilde{B}\left(-\partial_{y}, t\right)\right) \tilde{P}^{\prime}(x, y, t) \hat{F}_{\lambda}^{+\prime}(x, y, t)\right\}
\end{gathered}
$$


where $\quad \tilde{P} \stackrel{\text { def }}{=} P+P_{\infty} \quad$ and $\quad \tilde{B}(\mu, t)=D(\mu) B(\mu, t) D^{-1}(\mu)$. Here and below

$$
\partial_{x}=\frac{\partial}{\partial x}, \quad \partial_{y} \equiv \frac{\partial}{\partial y} \text {. }
$$

Combining the relations (2.10) and (2.11) and taking into account the identity (2.12) we find ${ }^{2}$

$$
\begin{gathered}
\int_{-\infty}^{+\infty} d x d y\left[\check { F } _ { \tilde { \lambda } } ^ { + } ( x , y , t ) \left(\tilde{B}\left(-\partial_{y} t\right) \tilde{P}^{\prime}(x, y, t) \hat{F}_{\lambda}^{+{ }^{\prime}}(x, y, t)\right.\right. \\
\left.\left.-\tilde{P}(x, y, t) \tilde{B}\left(-\partial_{y}, t\right) \hat{F}_{\lambda}^{+{ }^{\prime}}(x, y, t)\right)\right\}_{F}=0,
\end{gathered}
$$

where $\left(\Phi_{F}\right)_{\alpha \beta} \stackrel{\text { def }}{=} \Phi_{\alpha \beta}-\delta_{\alpha \beta} \Phi_{\alpha \alpha}(\alpha, \beta=1, \ldots, N M)$ for an arbitrary $N M \times N M$ matrix $\Phi$.

Let us represent the block diagonal matrix $B$ in the form

$$
B(\lambda, t)=\sum_{k=0}^{N-1} B_{k}\left(\lambda^{N}, t\right) \bar{A}^{k},
$$

where $B_{k}\left(\lambda^{N}, t\right)$ are scalar functions and $\bar{A}^{0} \stackrel{\text { def }}{=} I_{N M}$. Correspondingly for $\widetilde{B}\left(-\partial_{y}, t\right)$, we have

$$
\tilde{B}\left(-\partial_{y}, t\right)=\sum_{k=0}^{N-1} B_{k}\left(-\partial_{y}, t\right)\left(-A \partial_{y}+P_{\infty}\right)^{k} .
$$

It is easy to see that

$$
\left(-A \partial_{y}+P_{\infty}\right)^{k}=-\left(P_{\infty}^{\tau}\right)^{N-k} \partial_{y}+P_{\infty}^{k}
$$

where the symbol $\tau$ denotes a transposition of $M \times M$ blocks in $N M \times N M$ matrices.

We consider only functions $B_{k}\left(-\partial_{y}, t\right)$ which are entire on the first argument, i.e. $B_{k}\left(-\partial_{y}, t\right)=\sum_{n=0}^{n} B_{k n}(t)\left(-\partial_{y}\right)^{n}$, where $B_{k n}(t)$ are arbitrary functions. By virtue of $(2.14)$ and (2.15), for such functions $B_{k}\left(-\partial_{y}, t\right)$ the equality $(2.13)$ is equivalent to the equality

$$
\begin{aligned}
& \int_{-\infty}^{+\infty} d x d y \sum_{k=0}^{N-1} \sum_{n=0}^{\infty} B_{k n}(t) \operatorname{tr}\left\{\left(P_{\infty}^{\tau}\right)^{N-k} \tilde{P}^{\prime}(x, y, t) \breve{\Phi}_{(n+1)}^{(\alpha \beta)}(x, y, t)\right. \\
& \quad+P_{\infty}^{k} \widetilde{P}^{\prime}(x, y, t) \breve{\Phi}_{(n)}^{(\alpha \beta)}(x, y, t) \\
& \quad-\tilde{P}(x, y, t)\left(P_{\infty}^{\tau}\right)^{N-k}(-1)^{n+1} \breve{\Phi}_{(n+1)}^{(\alpha \beta)}(x, y, t) \\
& \left.\quad-\tilde{P}(x, y, t) P_{\infty}^{k}(-1)^{n} \hat{\Phi}_{(n)}^{(\alpha \beta)}(x, y, t)\right\}=0,(\alpha \neq \beta),
\end{aligned}
$$

where tr denotes a usual matrix trace and

$$
\left(\hat{\Phi}_{(n)}^{(\alpha \beta)}(x, y, t)\right)_{\gamma \delta} \stackrel{\text { def }}{=}\left(\hat{F}_{\tilde{\lambda}}^{+}(x, y, t)\right)_{\gamma \beta} \frac{\partial^{n}\left(\check{F}_{\tilde{\lambda}}^{+}(x, y, t)\right)_{\alpha \delta}}{\partial y^{n}},
$$

2 We omit some intermediate calculations which are typical for the generalised AKNS-technique (see e.g. $[8,13,14])$ 


$$
\begin{gathered}
\left(\hat{\Phi}_{(n)}^{(\alpha \beta)}(x, y, t)\right)_{\gamma \delta} \stackrel{\text { def }}{=} \frac{\partial^{n}\left(\hat{F}_{\lambda}^{+{ }^{\prime}}(x, y, t)\right)_{\gamma \beta}}{\partial y^{n}}\left(\hat{F}_{\bar{\lambda}}^{+}(x, y, t)\right)_{\alpha \delta} \\
(\alpha, \beta, \gamma, \delta=1, \ldots, N M ; \quad n=0,1,2, \ldots,) .
\end{gathered}
$$

\section{Recursion Operators}

For the further transformation of the equality (2.16) one must establish the relations between the expressions $\breve{\Phi}_{(n)}$ and $\widehat{\Phi}_{(n)}$ for different $n$, i.e. one must calculate the recursion operators.

Let us consider first the expression $\hat{\Phi}_{(n)}^{(\alpha \beta)}$. From Eqs. (2.1) and (2.3) we obtain

$$
\begin{aligned}
& \partial_{x} \hat{\Phi}_{(n)}^{(\alpha \beta)}+\partial_{y} \hat{\Phi}_{(n)}^{(\alpha \beta)} A=-\left[A, \hat{\Phi}_{(n+1)}^{(\alpha \beta)}\right] \\
& \quad+\hat{\Phi}_{(n)}^{(\alpha \beta)} P(x, y)-\sum_{m=0}^{n} C_{m}^{n} P_{(n-m)}^{\prime} \hat{\Phi}_{(m)}^{(\alpha \beta)} \quad(n=0,1,2, \ldots,),
\end{aligned}
$$

where $C_{m}^{n}=\frac{n !}{m !(n-m) !}$ and $P_{(k)}^{\prime} \stackrel{\text { def }}{=} \frac{\partial^{k} P(x, y, t)}{\partial y^{k}}$.

The relations (3.1) allow us to express all matrix elements of the quantity $\hat{\Phi}_{(n)}^{(\alpha \beta)}$ through $N-1$ independent ones.

Let us introduce the projection operation $\Delta_{k}:\left(\Phi_{\Delta_{k}}\right)_{i \ell} \stackrel{\text { def }}{=} \delta_{\ell k} \Phi_{i k}$. Applying the operations $\Delta_{k}$ to Eqs. (3.1) and taking into account the properties of the matrices $A, P_{\infty}, \hat{P}$, one obtains

$$
\begin{aligned}
& \partial_{x} \hat{\Phi}_{(n) \Delta_{1}}^{(\alpha \beta)}+\partial_{y} \hat{\Phi}_{(n) \Delta_{N}}^{(\alpha \beta)} A=-A \hat{\Phi}_{(n+1) \Delta_{1}}^{(\alpha \beta)} \\
& \quad+\hat{\Phi}_{(n+1) \Delta_{N}}^{(\alpha \beta)} A+\left(\hat{\Phi}_{(n) \Delta_{N}}^{(\alpha \beta)} \tilde{P}\right)_{\Delta_{1}}-\sum_{m=0}^{n} C_{m}^{n} P_{(n-m)}^{\prime} \hat{\Phi}_{(m) \Delta_{1}}^{(\alpha \beta)}, \\
& \partial_{x} \hat{\Phi}_{(n) \Delta_{k}}^{(\alpha \beta)}=-A \hat{\Phi}_{(n+1) \Delta_{k}}^{(\alpha \beta)}+\left(\hat{\Phi}_{(n) \Delta_{N}}^{(\alpha \beta)} \tilde{P}\right)_{\Delta_{k}} \\
& \quad-\hat{\Phi}_{(n) \Delta_{k-1}}^{(\alpha \beta)} P_{\infty}-\sum_{m=0}^{n} C_{m}^{n} P_{(n-m)}^{\prime} \hat{\Phi}_{(m) \Delta_{k}}^{(\alpha \beta)}, \quad(k=2,3, \ldots, N) .
\end{aligned}
$$

The relations (3.2) and (3.3) can be rewritten in a more compact form. Let us introduce the matrix infinite order triangular operators $\mathscr{T}$ and $T$ with matrix elements

$$
\begin{array}{ll}
\mathscr{T}_{(n, m)}=-\delta_{n m} \partial_{x}-C_{m}^{n} P_{(n-m)}^{\prime}, & n \geqq m, \\
\mathscr{T}_{(n, m)}=0, \quad m \geqq n+1 &
\end{array}
$$

and

$$
T_{(n, m)}=\delta_{m, n+1} I_{N M} . \quad(n, m=0,1,2, \ldots,)
$$

Matrix operators $\mathscr{T}$ and $T$ act on the infinite-component column 
$\Psi \stackrel{\text { def }}{=}\left(\hat{\Phi}_{(0)}, \hat{\Phi}_{(1)}, \hat{\Phi}_{(2)}, \ldots,\right)^{\tau}$ by the usual rules. For example,

$$
\begin{aligned}
\left(\mathscr{T}^{2} \Psi\right)_{(n)} & =\sum_{m_{1}=0}^{\infty} \mathscr{T}_{\left(n, m_{1}\right)} \sum_{m_{2}=0}^{\infty} \mathscr{T}_{\left(m_{1}, m_{2}\right)} \Phi_{\left(m_{2}\right)} \\
& =\sum_{m_{1}=0}^{n} \sum_{m_{2}=0}^{m_{1}} \mathscr{T}_{\left(n, m_{1}\right)} \mathscr{T}_{\left(m_{1}, m_{2}\right)} \Phi_{\left(m_{2}\right)} ; \quad(T \Psi)_{(n)}=\Phi_{(n+1)} .
\end{aligned}
$$

With the use of the operators $\mathscr{T}$ and $T$, the relations (3.2) and (3.3) can be represented in the form

$$
\begin{aligned}
(\mathscr{T}-A T) \Psi_{\Delta_{1}}^{(\alpha \beta)}= & \partial_{y} \Psi_{\Delta N}^{(\alpha \beta)} A+T \Psi_{\Delta_{N}}^{(\alpha \beta)} A-\left(\Psi_{\Delta_{N}}^{(\alpha \beta)} \tilde{P}\right)_{\Delta_{1}}, \\
\Psi_{\Delta_{k}}^{(\alpha \beta)}= & (\mathscr{T}-A T) \Psi_{\Delta_{k+1}}^{(\alpha \beta)}\left(A^{\tau}+P_{\infty}^{\tau}\right) \\
& +\left(\Psi_{\Delta_{N}}^{(\alpha \beta)} \tilde{P}\right)_{\Delta_{k+1}}\left(A^{\tau}+P_{\infty}^{\tau}\right), \quad(k=1,2, \ldots, N-1) .
\end{aligned}
$$

From the recurrence relations (3.7), we find

$$
\begin{aligned}
\Psi_{\Delta_{k}}^{(\alpha \beta)}= & (\mathscr{T}-A T)^{N-k} \Psi_{\Delta_{N}}^{(\alpha \beta)}\left(A^{\tau}+P_{\infty}^{\tau}\right)^{N-k}+ \\
& +\sum_{\ell=0}^{N-k-1}(\mathscr{T}-A T)^{\ell}\left(\Psi_{\Delta_{N}}^{(\alpha \beta)}\right)_{\Delta_{k+l+1}}\left(A^{\tau}+P_{\infty}^{\tau}\right)^{l+1}, \quad(k=1,2, \ldots, N-1) .
\end{aligned}
$$

By virtue of (3.8) one can express the quantity $\Psi_{\Delta_{1}}^{(\alpha \beta)}$ through $\Psi_{\Delta N}^{(\alpha \beta)}$. Substituting this expression for $\Psi_{\Delta_{1}}^{(\alpha \beta)}$ into (3.6) and taking into account the identity

$$
\left(\Phi_{\Delta_{N}} \tilde{P}\right)_{\Delta_{\ell}}\left(A+P_{\infty}\right)^{\ell}=-\Phi_{\Delta_{N}} \circ V_{\ell-1},
$$

where $\left(\Phi_{(n)} \circ V_{k}\right)_{l \ell} \stackrel{\text { def }}{=}\left(\Phi_{(n)}\right)_{i \ell} V_{k}$, we obtain

$$
\sum_{\ell=0}^{N}(\mathscr{T}-A T)^{\ell} \Psi_{\Delta N}^{(\alpha \beta)} \circ V_{\ell}=T \Psi_{\Delta N}^{(\alpha \beta)}-\partial_{y} \Psi_{\Delta N}^{(\alpha \beta)}
$$

Let us note that by virtue of the properties of the matrices $A, P_{\infty}$ and $\tilde{P}$ (for example, $A^{2}=A \widetilde{P}=\widetilde{P} A=A P_{\infty} \tilde{P}=\tilde{P} P_{\infty} A=0$ ) the operators $\left.\mathscr{T}-A T\right)^{\ell}$ are linear functions on the operator $T$, i.e.

$$
(\mathscr{T}-A T)^{\ell}=-\sum_{k_{1}+k_{2}=\ell-1} \mathscr{T}^{k_{1}} A T \mathscr{T}^{k_{2}}=r_{\ell} T+\mathscr{T}^{\ell}, \quad\left({ }^{\ell}=1,2,3, \ldots,\right)
$$

where

$$
r_{\ell}=-\sum_{k_{1}+k_{2}=\ell-1} \tilde{\mathscr{T}}^{k_{1}} A \tilde{\mathscr{T}}^{k_{2}}
$$

and

$$
\tilde{\mathscr{T}} \stackrel{\text { def }}{=} \mathscr{T}_{n n}=-\partial_{x}-P^{\prime}
$$

Substitution of (3.10) into (3.9) gives

$$
\sum_{\ell=0}^{N} r_{\ell}\left(T \Psi_{\Delta N}^{(\alpha \beta)} \circ V_{\ell}\right)-T \Psi_{\Delta N}^{(\alpha \beta)}=-\sum_{\ell=0}^{N} \mathscr{T}^{\ell}\left(\Psi_{\Delta N}^{(\alpha \beta)} \circ V_{\ell}\right)-\partial_{y} \Psi_{\Delta N}^{(\alpha \beta)}
$$

The equality (3.11) gives $N$ relations between $N$ expressions 
$\Psi_{1 N}^{(\alpha \beta)}, \Psi_{2 N}^{(\alpha \beta)}, \ldots, \Psi_{N N}^{(\alpha \beta)}$. The first nontrivial equation from (3.11) allows us to express $\Psi_{N N}^{(\alpha \beta)}$ through $\Psi_{1 N}^{(\alpha \beta)}, \ldots, \Psi_{N-1, N}^{(\alpha \beta)}$ :

$$
\Psi_{N N}^{(\alpha \beta)}(x, y, t)=\sum_{k=1}^{N-1} \ell_{k} \Psi_{N N}^{(\alpha \beta)}+\Psi_{N N}^{(\alpha \beta)}(x=+\infty, y, t),
$$

where

$$
\ell_{k} \cdot=\frac{1}{N} \sum_{l=1}^{N} \partial_{x}^{-1}\left(\left(\mathscr{T}^{l}\right)_{1 k}\left(. \circ V_{l}\right)\right)+\frac{1}{N} \partial_{x}^{-1} \partial_{y} \delta_{k 1},
$$

and $\left(\partial_{x}^{-1} f\right)(x, y) \stackrel{\text { def }}{=}-\int_{x}^{\infty} d x^{\prime} f\left(x^{\prime}, y\right)$.

Formula (3.12) contains the inhomogeneous term $\Psi_{N N}^{(\alpha \beta)}(x=+\infty, y, t)$. Similar inhomogeneous terms (namely $\left.\left(\Psi_{(x=+\infty, y, t)}^{(\alpha \beta)}\right)_{k \ell}\right)$ will appear after integration in further calculations too. Taking into account (2.5) and (2.6), one can show that $(\lambda>0)$

$$
\begin{aligned}
& \left(\hat{\Phi}_{(m)}^{(i n)}(x=+\infty, y, t)\right)_{k \ell}= \\
& \quad-\lambda^{N m} D_{k n} D_{i \ell}^{-1} \lim _{x \rightarrow+\infty} \exp \left\{\lambda\left(q^{n-1}-q^{i-1}\right) x\right\} .
\end{aligned}
$$

Let us denote by $\Psi^{(*)}$ the subspace of quantities $\Psi^{(i n)}$ for which $\operatorname{Re}\left(q^{n-1}-q^{i-1}\right)=$ $\cos ((2 \pi / N)(n-1))-\cos ((2 \pi / N)(i-1))<0$. For the indices $n$ and $i$ which satisfy this inequality one has $\lim _{x \rightarrow+\infty} \exp \left\{\lambda\left(q^{n-1}-q^{i-1}\right) x\right\}=0$. Therefore in the relations which contain the expression $\Psi^{(*)}$, the inhomogeneous terms will be absent. In particular, instead of (3.12) we have

$$
\Psi_{N N}^{(*)}=\sum_{k=1}^{N-1} \ell_{k} \Psi_{k N}^{(*)}
$$

By virtue of (3.14) one finds

$$
\Psi_{\Delta N}^{(*)}=M \Psi_{\Delta}^{(*)},
$$

where

$$
M=\left(\begin{array}{ccccc}
I_{M} & 0 & \ldots & & 0 \\
0 & I_{M} & \ldots & & 0 \\
\ldots & \ldots & \ldots & \ldots & \ldots \\
0 & 0 & \ldots & I_{M} & 0 \\
\ell_{1} & \ell_{2} & \ldots & \ell_{N-1} & 0
\end{array}\right), \Psi_{\Delta}=\left(\begin{array}{cccc}
0 & \ldots & 0 & \Psi_{1 N} \\
0 & \ldots & 0 & \Psi_{2 N} \\
\ldots & \ldots & \ldots & \ldots \\
0 & \ldots & 0 & \Psi_{N-1, N} \\
0 & \ldots & 0 & 0
\end{array}\right)
$$

Substituting (3.15) into the relation (3.11) we obtain

$$
\sum_{\ell=0}^{N} r_{\ell} T\left(M \Psi_{\Delta}^{(*)} \circ V_{\ell}\right)-T M \Psi_{\Delta}^{(*)}=-\sum_{\ell=0}^{N} \mathscr{T}^{\ell}\left(M \Psi_{\Delta}^{(*)} \circ V_{\ell}\right)-\partial_{y} M \Psi_{\Delta}^{(*)}
$$

Let us now rewrite the equality (3.17) directly in components $\hat{\Phi}_{(n) \Delta}^{(*)}$ of the infinitecomponent column $\Psi_{\Delta}^{(*)}$. Taking into account the explicit forms of the operators 
$\mathscr{T}$ and $T$ (see (3.4) and (3.5)), one finds

$$
\mathscr{G} \hat{\Phi}_{(n+1) \Delta}^{(*)}=\mathscr{F} \hat{\Phi}_{(n) \Delta}^{(*)}+\sum_{m=0}^{n-1} \mathscr{F}_{(n, m)} \hat{\Phi}_{(m) \Delta}^{(*)}, \quad(n=0,1,2, \ldots,)
$$

where

$$
\begin{aligned}
& \mathscr{G} \cdot \stackrel{\text { def }}{=} \sum_{\ell=0}^{N} r_{\ell}\left(\tilde{M} \cdot \circ V_{\ell}\right)-\tilde{M} \cdot \\
& \mathscr{F} \cdot \stackrel{\text { def }}{=} \sum_{\ell=0}^{N} \tilde{T}^{\ell}\left(\tilde{M} \cdot \circ V_{\ell}\right)-\partial_{y} \tilde{M} \cdot \\
& \mathscr{F}_{(n, m)} \stackrel{\text { def }}{=}-\sum_{\ell=0}^{N} \sum_{m^{\prime}=0}^{n}\left(\mathscr{T}^{\ell}\right)_{\left(n, m^{\prime}\right)}\left(M_{\left(m^{\prime}, m\right)} \cdot \circ V_{\ell}\right) .
\end{aligned}
$$

Operator $\tilde{M}$ is of the form (3.16) where instead of $\ell_{k}$ one must put the operators $\tilde{\ell_{k}}$, which are

$$
\tilde{\ell}_{k} \cdot=\frac{1}{N} \sum_{\ell=1}^{N} \partial_{x}^{-1}\left(\tilde{\mathscr{T}}^{\ell}\right)_{1 k}\left(\cdot \circ V_{\ell}\right)+\frac{1}{N} \delta_{1 k} \partial_{x}^{-1} \partial_{y}
$$

The relations (3.18) contain only independent expressions $\hat{\Phi}_{\Delta}^{(*)}$. Let us introduce the $N-1$-component column $\hat{\chi}_{(n)}=\left(\hat{\Phi}_{(n) 1 N}^{(*)}, \hat{\Phi}_{(n) 2 N}^{(*)}, \ldots, \hat{\Phi}_{(n) N-1, N}^{(*)}\right)^{\tau}$. In the terms of these quantities, the relation (3.18) is

$$
\tilde{\mathscr{G}} \hat{\chi}_{(n+1)}=\tilde{\mathscr{F}} \hat{\chi}_{(n)}+\sum_{m=0}^{n-1} \tilde{\mathscr{F}}_{(n, m)} \hat{\chi}_{(m)},
$$

where operators $\tilde{\mathscr{G}}, \widetilde{\mathscr{F}}, \tilde{\mathscr{F}}_{(n, m)}$ are block matrices of the order $N-1$. Their matrix elements are

$$
\begin{aligned}
\tilde{\mathscr{G}}_{i k} \cdot= & \sum_{l=0}^{N}\left(r_{\ell}\right)_{l+1, k}\left(\cdot \circ V_{\ell}\right)-\delta_{k, i+1} I_{M}, \\
\tilde{\mathscr{F}}_{i k} \cdot= & -\sum_{\ell=0}^{N}\left\{\left(\tilde{\mathscr{T}}^{\ell}\right)_{l+1, k}\left(\cdot \circ V_{t}\right)+\left(\tilde{\mathscr{T}}^{\ell}\right)_{i N}\left(\tilde{\ell}_{k} \cdot\right) \circ V_{\ell}\right\} \\
& -\delta_{k, i+1} I_{M} \partial_{y}-\delta_{N k} \partial_{y} \tilde{\ell}_{k}, \\
\left(\tilde{\mathscr{F}}_{(n, m)}\right)_{i k} \cdot= & -\sum_{l=0}^{N}\left\{\left(\mathscr{T}_{(n, m)}^{\ell}\right)_{i+1, k}\left(\cdot \circ V_{\ell}\right)\right. \\
& \left.+\sum_{l=0}^{N} \sum_{m^{\prime}=0}^{n}\left(\mathscr{T}_{\left(n, m^{\prime}\right.}^{l}\right)_{i N}\left(\left(\ell_{k}\right)_{\left(m^{\prime}, m\right)} \cdot\right) \circ V_{\ell}\right\} . \quad(i, k=1, \ldots, N-1) .
\end{aligned}
$$

It is not difficult to show that the operator $\tilde{\mathscr{G}}$ is a lower-triangular one $\left(\tilde{\mathscr{G}}_{i i}=-N \partial_{x}\right.$, $i=1, \ldots, N-1)$ and it has no nontrivial kernel. As a result, from (3.21) we have

$$
\hat{\chi}_{(n+1)}=\widetilde{\mathscr{G}}^{-1} \tilde{\mathscr{F}}_{\chi_{(n)}}+\sum_{m=0}^{n-1} \tilde{\mathscr{G}}^{-1} \tilde{\mathscr{F}}_{(n, m)} \check{\chi}_{(m)}, \quad(n=0,1,2, \ldots,) .
$$


From the relations (3.23) it follows that the recursion operators $\hat{\Lambda}_{n}$ exist such that

$$
\hat{\chi}_{(n)}=\hat{\Lambda}_{n} \chi_{(0)}, \quad(n=1,2,3, \ldots,,) .
$$

The operators $\hat{\Lambda}_{n}$ are calculated by the recurrence relations

$$
\begin{aligned}
\hat{\Lambda}_{n+1} & =\hat{\Lambda}_{1} \hat{\Lambda}_{n} \cdot+\sum_{m=0}^{n-1} \tilde{\mathscr{G}}^{-1} \tilde{\mathscr{F}}_{(n, m)} \hat{\Lambda}_{m}, \\
\hat{\Lambda}_{1} & =\tilde{\mathscr{G}}^{-1} \tilde{\mathscr{F}}, \quad \hat{\Lambda}_{0} \equiv I_{N M},
\end{aligned}
$$

where the operators $\tilde{\mathscr{G}}, \tilde{\mathscr{F}}_{\text {and }} \tilde{\mathscr{F}}_{(n, m)}$ are given by the formulas (3.22).

The operators $\hat{\Lambda}_{n}$ are just the recursion operators which we are interested in.

In an analogous manner one can show that

$$
\check{\chi}_{(n)}=\check{\Lambda}_{n} \chi_{(0)}, \quad(n=1,2,3, \ldots,),
$$

where recursion operators $\check{\Lambda}_{n}$ are calculated by the recurrence relations analogous to (3.25). It is easy also to show that

$$
\check{\Lambda}_{n}=\sum_{k=0}^{n}(-1)^{k} C_{k}^{n} \partial_{y}^{n-k} \hat{\Lambda}_{k} .
$$

In the further constructions we will also need the operators $\hat{\Lambda}_{n}^{+}$and $\check{\Lambda}_{n}^{+}$adjoint to the operators $\hat{\Lambda}_{n}$ and $\breve{\Lambda}_{n}$ with respect to the bilinear form

$$
\ll \chi^{\prime} \chi \gg=\int_{-\infty}^{+\infty} d x d y \operatorname{tr}\left(\chi^{\prime \tau}(x, y) \chi(x, y)\right),
$$

where $\chi$ and $\chi^{\prime}$ are columns with $N-1$ components. The recurrence relations for calculation of the operators $\hat{\Lambda}_{n}^{+}$are of the form

$$
\begin{aligned}
\hat{\Lambda}_{n+1}^{+} \cdot & =\hat{\Lambda}_{n}^{+} \hat{\Lambda}_{1}^{+} \cdot+\sum_{m=0}^{n-1} \hat{\Lambda}_{m}^{+} \tilde{\mathscr{F}}_{(n, m)}^{+}\left(\tilde{\mathscr{G}}^{+}\right)^{-1}, \\
\hat{\Lambda}_{1}^{+} & =\tilde{\mathscr{F}}^{+}\left(\tilde{\mathscr{G}}^{+}\right)^{-1}, \quad(n=1,2,3, \ldots),
\end{aligned}
$$

where matrix elements of the operators $\tilde{\mathscr{G}}^{+}, \tilde{\mathscr{F}}^{+}$and $\tilde{\mathscr{F}}_{(n, m)}^{+}$are

$$
\begin{aligned}
\tilde{\mathscr{G}}_{i k}^{+} \cdot= & \sum_{\ell=0}^{N} V_{\ell}\left(r_{\ell}^{+}\right)_{i, k+1}-\delta_{i, k+1} I_{M}, \\
\tilde{\mathscr{F}}_{i k}^{+} \cdot= & -\sum_{\ell=0}^{N} V_{\ell}\left(\tilde{\mathscr{T}}^{+}\right)_{i, k+1}^{\ell}+\delta_{k N} \tilde{\ell}_{k}^{+} \partial_{y} \\
& -\sum_{\ell=0}^{N} \tilde{\ell}_{i}^{+} V_{\ell}\left(\tilde{\mathscr{T}}^{+}\right)_{N k}^{l}+\delta_{i, k+1} I_{M} \partial_{y}, \\
\left(\tilde{\mathscr{F}}_{(n, m)}^{+}\right)_{i k} \cdot= & -\sum_{\ell=0}^{N} V_{\ell}\left(\left(\mathscr{T}^{+}\right)_{(n, m)}^{\ell}\right)_{i, k+1}-\sum_{\ell=0}^{N} \sum_{m^{\prime}=0}^{n}\left(\ell_{i}^{+}\right)_{\left(m^{\prime}, m\right)}\left(\left(\mathscr{T}^{+}\right)_{\left(n, m^{\prime}\right)}^{\ell}\right)_{N k},
\end{aligned}
$$

and

$$
\begin{aligned}
\left(\ell_{i}^{+}\right)_{(n, m)} & =\frac{1}{N} \sum_{\ell=1}^{N} V_{\ell}\left(\left(\mathscr{T}^{+}\right)_{(n, m)}^{\ell}\right)_{k 1} \partial_{x}^{-1}+\frac{1}{N} \delta_{k 1} \delta_{(n, m)} \partial_{y} \partial_{x}^{-1}, \\
\tilde{\ell}_{i}^{+} \cdot & =\frac{1}{N} \sum_{\ell=1}^{N} V_{\ell}\left(\tilde{\mathscr{T}}^{+}\right)_{k 1}^{\ell} \partial_{x}^{-1}+\frac{1}{N} \delta_{k 1} \partial_{y} \partial_{x}^{-1} .
\end{aligned}
$$


In the formulas (3.30) and in all adjoint operators (marked by +$)\left(\partial_{x}^{-1} f\right)$ $(x, y)=\int_{-\infty}^{x} d x^{\prime} f\left(x^{\prime}, y\right)$.

The operators $r_{\ell}^{+}$are calculated by the formulas

$$
r_{\ell}^{+}=\sum_{k_{1}+k_{2}=\ell-1}\left(\tilde{\mathscr{T}}^{+}\right)^{k_{1}} A^{\tau}\left(\tilde{\mathscr{T}}^{+}\right)^{k_{2}}
$$

where $\mathscr{T}^{+} \stackrel{\text { def }}{=} \mathscr{T}_{n n}^{+}$and

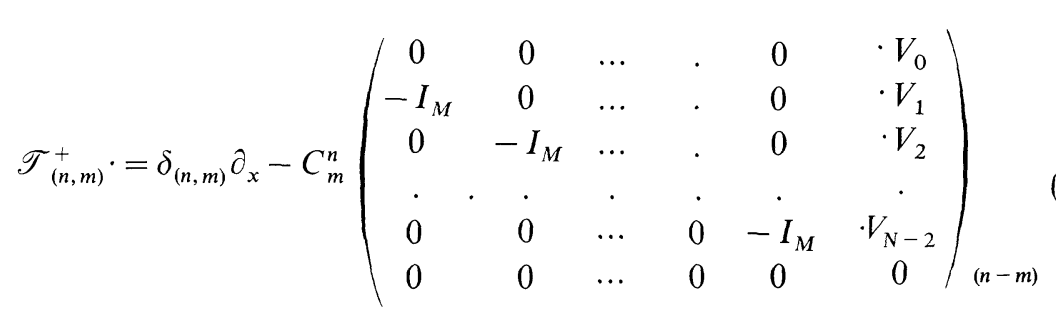

Formulas (3.28)-(3.32) give a somewhat cumbersome but direct method for calculation of the recursion operators $\hat{\Lambda}_{n}^{+}$.

The operators $\check{\Lambda}_{n}^{+}$can be found by the recurrence relations analogous to (3.28) or by the formula

$$
\check{\Lambda}_{n}^{+}=(-1)^{n} \sum_{k=0}^{n} C_{k}^{n} \hat{\Lambda}_{k}^{+} \partial_{y}^{n-k} .
$$

\section{General Form of the Integrable Equations}

In the previous section it was shown that matrix elements of the matrices $\check{\Phi}_{(n)}^{(*)}, \Phi_{(n)}^{(*)}$ can be expressed through $\hat{\chi}_{(m)}$ and $\check{\chi}_{(m)}$. So let us transform the equality (2.16) into a form which contains only independent quantities $\hat{\chi}$ and $\check{\chi}$.

Taking into account the properties of the matrices $P_{\infty}$ and $\tilde{P}$, one can show that the equality (2.16) is equivalent to the following one

$$
\begin{gathered}
\int_{-\infty}^{+\infty} d x d y \sum_{k=0}^{N-1} \sum_{n=0}^{\infty} b_{k n}(t) \operatorname{tr}\left\{P_{\infty}^{k} \tilde{P}^{\prime}(x, y, t) \Phi_{(n) \Delta_{N-k}}^{(*)}(x, y, t)-\right. \\
\left.-\tilde{P}(x, y, t)(-1)^{n+1}\left(P_{\infty}^{\tau}\right)^{N-k} \widehat{\Phi}_{(n+1) \Delta_{N}}^{(*)}(x, y, t)-\tilde{P}(x, y, t)(-1)^{n} P_{\infty}^{k} \hat{\Phi}_{(n) \Delta_{N}}^{(*)}(x, y, t)\right\}=0 .
\end{gathered}
$$

From the relation analogous to (3.8) we find

$$
\begin{aligned}
\Phi_{(n) \Delta_{N-K}}^{(*)} & =\left(\partial_{x}+A \partial_{y}+P^{\prime}-A T\right)^{N-k} \breve{\Phi}_{(n) \Delta_{N}}^{(*)}\left(A^{\tau}+P_{\infty}^{\tau}\right)^{N-k} \\
& -\sum_{l=0}^{N-k-1}\left(\partial_{x}+A \partial_{y}+P^{\prime}-A T\right)^{\ell} \sum_{m=0}^{n} C_{m}^{n}\left(\check{\Phi}_{(m) \Delta_{N}}^{(*)} \tilde{P}_{(n-m)}\right)_{\Delta_{k}+\ell+1}\left(A^{\tau}+P_{\infty}^{\tau}\right)^{\ell+1} \\
& \stackrel{\text { def }}{=} \sum_{m} \breve{G}_{(n, m)}^{(k)} \check{\Phi}_{(m) \Delta N}^{(*)} .
\end{aligned}
$$


Passing on in Eq. (4.1) from the matrices $\Phi_{(n) \Delta N}^{(*)}$ to the columns $\chi_{(n)}$ and introducing the $N-1$-component column $V(x, y, t)=\left(V_{0}(x, y, t), V_{1}(x, y, t), \ldots\right.$, $\left.V_{N-2}(x, y, t)\right)^{\tau}$, we obtain

$$
\begin{aligned}
& \sum_{k=0}^{N-1} \sum_{n=0}^{\infty} b_{k n}(t) \ll V^{\prime \tau} \sum_{m} \check{G}_{(n, m)}^{(k)} \check{\chi}_{(m)} \\
& \quad-(-1)^{n+1} V^{\tau} \tilde{K}^{(k)} \hat{\chi}_{(n+1)}-(-1)^{n} V^{\tau} \sum_{m} \hat{N}_{(n, m)}^{(k)} \hat{\chi}_{(m)} \gg=0
\end{aligned}
$$

where

$$
\begin{aligned}
\left(\widetilde{G}_{(n, m)}^{(k)}\right)_{i \ell \cdot} & =\left(\check{\mathscr{G}}_{(n, m)}^{(k)}\right)_{i \ell}+\sum_{m^{\prime}}\left(\check{\mathscr{G}}_{\left(n, m^{\prime}\right)}^{(k)}\right)_{i N}\left(\check{\ell}_{\ell}\right)_{\left(m^{\prime}, m\right)}, \\
\left(\tilde{K}^{(k)}\right)_{i \ell} & =\delta_{i, \ell+N-k} I_{M}, \\
\left(\tilde{N}_{(n, m)}^{(k)}\right)_{i \ell} & =\delta_{i, \ell-k} I_{M}+\delta_{i N-k}\left(\ell_{\ell}\right)_{(n, m)}, \quad(i, \ell=1, \ldots, N-1) .
\end{aligned}
$$

Operators $\check{\ell}_{k}$ are calculated analogously to the operators $\ell_{k}$.

Lastly, by virtue of (3.24) and (3.26), the equality (4.3) is equivalent to the equality

$$
\begin{aligned}
& \ll \chi_{(0)} \sum_{k=0}^{N-1} \sum_{n=0}^{\infty} b_{k n}(t)\left\{\sum_{m} \check{\Lambda}_{m}^{+} \tilde{G}_{(n, m)}^{(k)+} V^{\prime}\right. \\
& \left.-\left((-1)^{n+1} \hat{\Lambda}_{n+1}^{+} \tilde{K}^{(k)^{+}}+(-1)^{n} \sum_{m} \hat{\Lambda}_{m}^{+} \tilde{N}_{(n, m)}^{(k)}\right) V\right\} \gg=0,
\end{aligned}
$$

where operators $\hat{\Lambda}_{n}^{+}$and $\check{\Lambda}_{n}^{+}$are calculated by the formulas (3.28)-(3.33), and

$$
\begin{aligned}
\left(\tilde{G}_{(n, m)}^{(k)^{+}}\right)_{i \ell} & =\left(\check{\mathscr{G}}_{(n, m)}^{(k)+}\right)_{i \ell}+\sum_{m^{\prime}}\left(\breve{\ell}_{i}^{+}\right)_{\left(m^{\prime}, m\right)}\left(\breve{G}_{\left(n, m^{\prime}\right)}^{(k)^{+}}\right)_{N \ell}, \\
\left(\tilde{K}^{(k)^{+}}\right)_{i \ell} & =\delta_{i, \ell-N+k} I_{M}, \\
\left(\tilde{N}_{(n, m)}^{(k)^{+}}\right)_{i \ell} & =\delta_{i, \ell+k} I_{M}+\delta_{\ell, N-k}\left(\ell_{i}^{+}\right)_{(n, m)},(i, k, \ell=1, \ldots, N-1) .
\end{aligned}
$$

The equality (4.5) is fulfilled if

$$
\begin{aligned}
& \sum_{k=0}^{N-1} \sum_{n=0}^{\infty} b_{k n}(t)\left\{\sum_{m} \check{\Lambda}_{m}^{+} \tilde{G}_{(n, m)}^{(k)^{+}} V^{\prime}\right. \\
& \left.\quad-\left((-1)^{n+1} \hat{\Lambda}_{n+1}^{+} \tilde{K}^{(k)^{+}}+(-1)^{n} \sum_{m} \hat{\Lambda}_{m}^{+}\left(\tilde{N}^{(k)^{+}}\right)_{(n, m)}\right) V\right\}=0 .
\end{aligned}
$$

Thus, we have found the transformations of the potential $V \rightarrow V^{\prime}$ which correspond to the transformations of the scattering matrix $S \rightarrow S^{\prime}$ of the form (2.11). These transformations $V \rightarrow V^{\prime}$ are given by the relation (4.7), where $b_{k n}(t)$ are arbitrary functions.

It is not difficult to show that the transformations (2.11), (4.7) form an infinitedimensional abelian group. The transformations from this group are characterised by $N$ functions $B_{k}\left(\lambda^{N}, t\right)$ which are entire on $\lambda^{N}$.

The infinite-dimensional abelian group of the transformations (2.11), (4.7) which 
act on the manifold of the potentials $\{V(x, y, t)\}$ by the formula (4.7) and on the manifold of the scattering matrices $\{\hat{S}(\tilde{\lambda}, \lambda, t)\}$ by the formula (2.11) plays a fundamental role in the analysis of the nonlinear systems connected with problem (1.1) and their group-theoretical properties.

The group of the transformations (2.11), (4.7) contains various types of transformations. Let us consider the infinitesimal displacement in time $t: t \rightarrow t^{\prime}=$ $t+\varepsilon, \varepsilon \rightarrow 0$. In this case

$$
\begin{gathered}
V^{\prime}(x, y, t)=V\left(x, y, t^{\prime}\right)=V(x, y, t)+\varepsilon \frac{\partial V(x, y, t)}{\partial t}, \\
B_{k}\left(\lambda^{N}, t\right)=\delta_{k 0}-\varepsilon \Omega_{k}\left(\lambda^{N}, t\right)=\delta_{k 0}-\varepsilon \sum_{n=0}^{\infty} \omega_{k n}(t) \lambda^{N n} .
\end{gathered}
$$

Substituting these expressions into (4.7) and keeping the terms of the first order on $\varepsilon$, we obtain

$$
\frac{\partial V(x, y, t)}{\partial t}+\mathscr{L}_{\Omega}\left(L^{+}, t\right) V=0
$$

where

$$
\begin{aligned}
& \mathscr{L}_{\Omega}\left(L^{+}, t\right)=\sum_{k=1}^{N-1} \sum_{n=0}^{\infty} \omega_{k n}(t)\left\{\left.\sum_{m} \check{L}_{m}^{+} \tilde{G}_{(n, m)}^{(k)^{+}}\right|_{V^{\prime}=V}\right. \\
& \left.-(-1)^{n+1} \hat{L}_{n+1}^{+} \tilde{K}^{(k)^{+}}-\left.(-1)^{n} \sum_{m} \hat{L}_{m}^{+}\left(\tilde{N}^{(k)^{+}}\right)_{(n, m)}\right|_{V^{\prime}=V}\right\},
\end{aligned}
$$

and $\left.L_{n}^{+} \stackrel{\text { def }}{=} \Lambda_{n}^{+}\right|_{V^{\prime}=V}$. The operators $\hat{L}_{n}^{+}$and $\check{L}_{n}^{+}$are calculated by formulas (3.28)-(3.33) at $V^{\prime}=V$.

For the scattering matrix $\hat{S}$ under infinitesimal time displacement $\hat{S}^{\prime}(\tilde{\lambda}, \lambda, t)=$ $\hat{S}(\tilde{\lambda}, \lambda, t)+\varepsilon \partial / \hat{S}(\tilde{\lambda}, \lambda, t) / \partial t$, and correspondingly from (2.11), one has

$$
\frac{\partial \hat{S}(\tilde{\lambda}, \lambda, t)}{\partial t}=Y(\tilde{\lambda}, t) \hat{S}(\tilde{\lambda}, \lambda, t)-\hat{S}(\tilde{\lambda}, \lambda, t) Y(\lambda, t)
$$

where $Y(\lambda, t)=\sum_{k=1}^{N-1} \Omega_{k}\left(\lambda^{N}, t\right) \bar{A}^{k}$.

Therefore we obtain nonlinear evolution equations in $1+2$ dimensions $(t, x, y)$ as the infinitesimal form of the transformations (4.7) generated by the time displacement.

The class of nonlinear equations (4.8) is characterised by arbitrary integers $N$ and $M$, by recursion operators $L_{n}^{+}$, and by arbitrary functions $\omega_{k n}(t)(k=1, \ldots, N-1)$. A choice of the concrete $N, M$ and functions $\omega_{k n}(t)$ leads to the concrete equation of the form (4.8). The case $N=2$ will be considered in Sect. 6 .

Nonlinear evolution equations (4.8) in $1+2$ dimensions are just the equations integrable by the IST method with the help of the two-dimensional problem (1.1). Using the two-dimensional version of the IST method (see $[15,16]$ ) one can find a broad class of the exact solutions of Eqs. (4.8).

In concluding this section let us attract attention to the fact that by virtue of 
(4.10) the diagonal elements $\hat{S}_{\alpha \alpha}(\lambda, \lambda)$ of the scattering matrix are time-independent: $d \widehat{S}_{a \alpha}(\lambda, \lambda) / d t=0$ for any functions $\Omega_{k}\left(\lambda^{N}, t\right)$. Therefore the quantities $\hat{S}_{a \alpha}(\lambda, \lambda)$ for any $\lambda$ are integrals of motion of Eqs. (4.8). If one expands $\operatorname{tr}\left(\bar{A}^{p} \ln \hat{S}_{D}(\lambda, \lambda)\right)\left(\left(S_{D}\right)_{\alpha \beta} \stackrel{\text { def }}{=} \delta_{\alpha \beta} S_{a \alpha}\right)$ in the asymptotic series on $\lambda^{-1}: \operatorname{tr}\left(\bar{A}^{p} \ln \hat{S}_{D}(\lambda, \lambda)\right)=\sum_{n=0}^{\infty} \lambda^{-n} C_{n}^{(p)}$, then one obtains a counting set of the integrals of motion $C_{n}^{(p)}(p=1, \ldots, N-1 ; n=0,1,2, \ldots$,$) . By$ standard procedure (see e.g. $[1,14]$ ), one can find the explicit dependence of the integrals of motion $C_{n}^{(p)}$ on $V_{0}(x, y, t), V_{1}(x, y, t), \ldots, V_{N-2}(x, y, t)$. Let us emphasize that these integrals of motion are universal one, i.e. they are integrals of motion for any equations of the form (4.8).

In conclusion let us consider the one-dimensional limit when all the potentials $V_{0}, V_{1}, \ldots, V_{N-2}$ in (1.1) are independent of the variable $y\left(\partial V_{k} / \partial y=0\right)$. If one performs the Fourier transform over the variable $y$, i.e. $\chi(x, y, t)=\int d \lambda$ $\exp \left(-\lambda^{N} y\right) \tilde{\chi}(x, \lambda, t)$, then problem (1.1) is reduced to the one dimensional Gelfand-Dickey spectral problem for $\tilde{\chi}(x, \lambda, t)$;

$$
\frac{\partial^{N} \tilde{\chi}}{\partial x^{N}}+V_{N-2}(x, t) \frac{\partial^{N-2} \tilde{\chi}}{\partial x^{N-2}}+\cdots+V_{0}(x, t) \tilde{\chi}=\lambda^{N} \tilde{x}
$$

In this one dimensional case $\hat{F}_{\lambda}^{ \pm}(x, y, t)=(2 \pi i)^{-1 / 2} \exp \left(-\lambda^{N} y\right) \hat{\tilde{F}}^{ \pm}(x, \lambda, t)$, $\check{F}_{\lambda}^{ \pm}(x, y, t)=(2 \pi i)^{-1 / 2} \exp \left(\lambda^{N} y\right) \tilde{\widetilde{F}}^{ \pm}(x, \lambda, t)$, and from the relations (2.5)-(2.9) it follows that $\widetilde{\widetilde{F}}=(\widetilde{\widetilde{F}})^{-1}$ and

$$
\hat{S}(\tilde{\lambda}, \lambda, t)=\delta(\tilde{\lambda}-\lambda) S(\lambda, t), \quad \check{S}(\tilde{\lambda}, \lambda, t)=\delta(\tilde{\lambda}-\lambda) S^{-1}(\lambda, t),
$$

where $S(\lambda, t)$ is the one dimensional scattering matrix.

Then for the quantities $\hat{\Phi}_{(n)}$ and $\breve{\Phi}_{(n)}$, we have $\hat{\Phi}_{(n)}=\left(-\lambda^{N}\right)^{n} \tilde{\Phi}(x, \lambda, t)$, $\breve{\Phi}_{(n)}=\left(\lambda^{N}\right)^{n} \tilde{\Phi}(x, \lambda, t)$. Furthermore in the case $\partial V_{k} / \partial y=0$, the operators $\tilde{\mathscr{F}}_{(n, m)}$ in (3.25) at $n \neq m$ are equal to zero, and therefore the relations (3.24) $-(3.26),(3.28)$ are reduced to the following ones: $-\lambda^{N} \tilde{\chi}(x, \lambda, t)=\hat{\Lambda}_{1} \tilde{\chi}(x, \lambda, t)$, and

$$
\begin{gathered}
\hat{\Lambda}_{n}=\left(\hat{\Lambda}_{1}\right)^{n}, \quad \hat{\Lambda}_{n}^{+}=\left(\hat{\Lambda}_{1}^{+}\right)^{n}, \\
\hat{\Lambda}_{n}=\left(-\hat{\Lambda}_{1}\right)^{n}, \quad \check{\Lambda}_{n}^{+}=\left(-\hat{\Lambda}_{1}^{+}\right)^{n} \quad(n=1,2,3 \ldots) .
\end{gathered}
$$

As a result, at $\partial V_{k} / \partial y=0,(k=0,1, \ldots, N-2)$, the transformations (4.7) and Eqs. (4.8) are reduced to the corresponding transformations and equations connected with the one dimensional Gelfand-Dickey problem (4.11) (see Ref. [13]).

\section{Transformation Properties of the Integrable Equations}

General transformation properties of Eqs. (4.8) are mainly analogous to those for the equations integrable by the problem $\partial \Psi / \partial x+A \partial \Psi / \partial y+P(x, y, t) \Psi=0[14]$. So we consider them briefly.

The group of transformations (2.11), (4.7) plays a main role in the analysis of the general group-theoretical properties of Eqs. (4.8).

Let us first consider the transformations (2.11), (4.7) with time-independent matrices $B \neq C$. These transformations form an infinite-dimensional abelian group 
and, as it is easy to see, does not change the evolution law (4.10) of the scattering matrix. Therefore they convert the solutions of the concrete equation of the form (4.8) into the solutions of the same equation, i.e. these transformations are autoBäcklund transformations for Eqs. (4.8).

The group of transformations (2.11), (4.7) also contains as a subgroup the infinite dimensional abelian symmetry group of Eqs. (4.8). In the infinitesimal form these symmetry transformations are $\left(V \rightarrow V^{\prime}=V+\delta V\right)$

$$
\begin{aligned}
\delta V= & -\sum_{k=1}^{N-1} \sum_{n=0}^{\infty} f_{k n}\left\{\left.\sum_{m} \check{L}_{m}^{+} \tilde{G}_{(n, m)}^{(k)+}\right|_{V^{\prime}=V}\right. \\
& \left.-(-1)^{n+1} \hat{L}_{n+1}^{+} \tilde{K}^{(k)^{+}}-\left.(-1)^{n} \sum_{m} \hat{L}^{+m} \tilde{N}_{(n, m)}^{(k)+}\right|_{V^{\prime}=V}\right\} V,
\end{aligned}
$$

where $f_{k n}$ are arbitrary constants. The transformations (5.1) are symmetry transformations for any equation of the form (4.8).

And finally, the transformations (2.11), (4.7) with time-dependent matrices $B$ and $C$ are generalised Bäcklund transformations: they convert the solutions of certain equations (4.8) into the solutions of the other equation (with other functions $\omega_{k n}(t)$ ) of the form (4.8).

\section{An Example $N=2$ : Nonstationary Schrödinger Spectral Problem}

Here we illustrate the general results of the previous sections. Problem (1.1) for $N=2$ is the nonstationary Schrödinger spectral problem $\partial \chi / \partial y+\partial^{2} \chi / \partial x^{2}$ $+V_{0}(x, y, t) \chi=0$. In the scalar case $M=1$ this problem was used for the integration of the Kadomtsev-Petviashvili equation $[15,17,18]$.

For $N=2$ the general equations (4.8) are of the form $\left(V_{0} \equiv U\right)$

$$
\begin{aligned}
& \frac{\partial U(x, y, y)}{\partial t}-\frac{1}{2} \sum_{n=0}^{\infty} \omega_{1 n}(t)\left\{\hat{L}_{n}^{+}\left(\partial_{y} \partial_{x}^{-1} U+\partial_{x} U+U \partial_{x}^{-1} U\right)\right. \\
& +(-1)^{n} \check{L}_{n}^{+}\left(-\partial_{y} \partial_{x}^{-1} U+\partial_{x} U+\left(\partial_{x}^{-1} U\right) U\right) \\
& \left.\quad-\sum_{m=0}^{n} C_{m}^{n}\left(\hat{L}_{m}^{+}\left(\partial_{x}^{-1} U\right) U_{(n-m)}+(-1)^{n} \check{L}_{m}^{+} U_{(n-m)} \partial_{x}^{-1} U\right)\right\}=0 .
\end{aligned}
$$

Recursion operators $\hat{L}_{n}^{+}$and $\breve{L}_{n}^{+}$are calculated by formulas (3.28)-(3.33) at $N=2$ $\left(U^{\prime}=U\right)$. For example

$$
\begin{aligned}
\hat{L}_{1}^{+} \cdot= & -\frac{1}{4}\left\{\left(\partial_{y} \partial_{x}^{-1}+\partial_{x}\right)^{2}+2[U(x, y), .]_{+}\right. \\
& +\left[\partial_{x} U(x, y), \partial_{x}^{-1} \cdot\right]_{+}-\left[U(x, y), \partial_{y} \partial_{x}^{-2} \cdot\right]_{-} \\
& \left.+\partial_{y} \partial_{x}^{-1}\left[U, \partial_{x}^{-1} \cdot\right]_{-}+\left[U(x, y), \partial_{x}^{-1}\left[U, \partial_{x}^{-1} \cdot\right]_{-}\right]_{-}\right\}
\end{aligned}
$$

and $\breve{L}_{1}^{+}=-\hat{L}_{1}^{+}-\partial_{y}$, where $[A, B]_{ \pm} \stackrel{\text { def }}{=} A B \pm B A$.

The simplest equation (6.1) corresponds to $\omega_{12}=\omega_{13}=\cdots=0$ and it has the 
form

$$
\begin{aligned}
& \frac{\partial U(x, y, t)}{\partial t}-\omega_{10}(t) \frac{\partial U(x, y, t)}{\partial x}+\frac{\omega_{11}(t)}{4}\left(\frac{\partial^{3} U(x, y, t)}{\partial x^{3}}+3 \int_{-\infty}^{x} d x^{\prime} \frac{\partial^{2} U\left(x^{\prime}, y, t\right)}{\partial y^{2}}\right. \\
&\left.+3 \frac{\partial}{\partial x}\left(U^{2}(x, y, t)\right)+3\left[U(x, y, t), \int_{-\infty}^{x} d x^{\prime} \frac{\partial U\left(x^{\prime}, y, t\right)}{\partial y}\right]_{-}\right)=0 .
\end{aligned}
$$

In the scalar case $(M=1)$ and $\omega_{10}=0, \omega_{11}=-4$, Eq. (6.3) is the well-known Kadomtsev-Petviashvili (KP) equation. For $M>1$ Eq. (6.3) is the matrix KP equation (see e.g. [19]). The KP equation (6.3) is the lowest one $\left(\mathrm{KP}_{1}\right)$ from the infinite family (KP family) of the $1+2$ dimensional equations (6.1): the $\mathrm{KP}_{n}$ equation corresponds to $\omega_{11}=\cdots=\omega_{1 n-1}=\omega_{1 n+1}=\cdots=0, \omega_{1 n}=-2^{2 n}$, $(n=1,2,3, \ldots)$.

The simplest Bäcklund transformation (BT) (4.7) corresponds to constant $b_{00}$ and $b_{10}$ and $b_{k n}=0,(k=0,1 ; n=1,2, \ldots$,$) , and it is B_{b}\left(U \rightarrow U^{\prime}\right)$ :

$$
\begin{aligned}
& b\left(U^{\prime}-U\right)+\frac{\partial}{\partial x}\left(U^{\prime}+U\right)-\int_{-\infty}^{x} d x^{\prime} \frac{\partial}{\partial y}\left(U^{\prime}\left(x^{\prime}, y, t\right)-U\left(x^{\prime}, y, t\right)\right) \\
& +\int_{-\infty}^{x} d x^{\prime}\left(U^{\prime}\left(x^{\prime}, y, t\right)-U\left(x^{\prime}, y, t\right)\right) U^{\prime}(x, y, t) \\
& -U(x, y, t) \int_{-\infty}^{x} d x^{\prime}\left(U^{\prime}\left(x^{\prime}, y, t\right)-U\left(x^{\prime}, y, t\right)\right)=0
\end{aligned}
$$

where $b=2 b_{00} / b_{10}$. Introducing a quantity $W(x, y, t)=\int_{-\infty}^{x} d x^{\prime} U\left(x^{\prime}, y, t\right)$, we obtain a local form of BT (6.4):

$b \frac{\partial}{\partial x}\left(W^{\prime}-W\right)+\frac{\partial^{2}}{\partial x^{2}}\left(W^{\prime}+W\right)-\frac{\partial}{\partial y}\left(W^{\prime}-W\right)+\left(W^{\prime}-W\right) \frac{\partial W^{\prime}}{\partial x}-\frac{\partial W}{\partial x}\left(W^{\prime}-W\right)=0$.

Let us emphasize that the BT (6.4) is a universal one, i.e. it is a BT for any equation of the form (6.1).

The BT (6.5) allows us to construct the infinite family of the solutions of Eqs. (6.1) by almost pure algebraic operations. Indeed, let us consider the following diagram

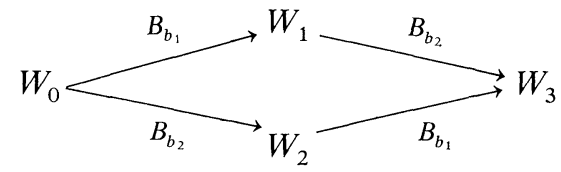

which expresses the commutativity of BT's (6.5): $B_{b_{1}} B_{b_{2}}=B_{b_{2}} B_{b_{1}}$. Here $W_{0}, W_{1}, W_{2}, W_{3}$ are four solutions of the concrete (but any) equation of the form (6.1). With the use of relation (6.5) for all four solutions $W_{0}, W_{1}, W_{2}, W_{3}$ from (6.6), we obtain

$$
\begin{aligned}
W_{3}= & \left(b_{1}-b_{2}+W_{1}-W_{2}\right)^{-1}\left\{\left(b_{1}-b_{2}\right)\left(W_{1}+W_{2}-W_{0}\right)\right. \\
& \left.-W_{0}\left(W_{1}+W_{2}\right)+W_{1}^{2}-W_{2}^{2}+2 \frac{\partial}{\partial x}\left(W_{1}-W_{2}\right)\right\} .
\end{aligned}
$$


Therefore given three solutions $W_{0}, W_{1}, W_{2}$, one can easily calculate the fourth solution $W_{3}$ by formula (6.7). Let us emphasize that relation (6.7) is a universal one, i.e. it is valid for all equations of the form (6.1) and in particular for any equation from the KP family.

Relation (6.7) is just the nonlinear superposition principle for Eqs. (6.1). Some concrete nonlinear superposition principles for some concrete $1+1$ dimensional equations (for example, for the Korteweg-de Vries equation) are well-known (see e.g. Ref. [20]).

In the scalar case $(M=1), \mathrm{BT}(6.5)$ and nonlinear superposition formula (6.7) are reduced to the following

$$
b\left(W^{\prime}-W\right)+\frac{\partial}{\partial x}\left(W^{\prime}+W\right)+\frac{1}{2}\left(W^{\prime}-W\right)^{2}-\int_{-\infty}^{x} d x^{\prime} \frac{\partial}{\partial y}\left(W^{\prime}\left(x^{\prime}, y\right)-W\left(x^{\prime}, y\right)\right)=0,
$$

and

$$
W_{3}=W_{1}+W_{2}-W_{0}+2 \frac{\partial}{\partial x} \ln \left(b_{1}-b_{2}+W_{1}-W_{2}\right)
$$

which coincide at $b=0$ with those found earlier by another method in Ref. [21].

Let us consider for definiteness the scalar $\mathrm{KP}_{1}$ equation (6.3). Let us start with the trivial solution $W_{0}=0$. If one applies $\mathrm{BT}$ (6.8) to this solution, then one obtains the solution $W_{1}$, which can be found from the equation

$$
b_{1} \frac{\partial W_{1}}{\partial x}+\frac{\partial^{2} W_{1}}{\partial x^{2}}-\frac{\partial W_{1}}{\partial y}+W_{1} \frac{\partial W_{1}}{\partial x}=0 .
$$

One of the solutions of Eq. (6.10) is the well-known soliton-type solution of the $\mathrm{KP}_{1}$ equation (see e.g. [1]):

$U(x, y, t)=\frac{\left(\frac{b_{1}}{2}-a\right)^{2}}{2}\left(\cosh \frac{\left(\frac{b_{1}}{2}-a\right) x+\left(\left(\frac{b_{1}}{2}\right)^{2}-a^{2}\right) y-4\left(\left(\frac{b_{1}}{2}\right)^{3}-a^{3}\right) t+c}{2}\right)^{-2}$

where $a$ and $c$ are arbitrary real constants and $b_{1}<0$.

Let us now take the trivial solution as $W_{0}$, and soliton-type solutions (6.11) with constants $b_{1}$ and $b_{2}$ as $W_{1}$ and $W_{2}$. Then using formula (6.9) we find the two-soliton solution $W_{3}$. An obvious proceeding of this procedure gives any $N$-soliton solution of the $\mathrm{KP}_{1}$ equation.

In the scalar case one can also obtain from (6.6) an other nonlinear superposition formula for BT (6.8). It has the form

$$
W_{3}=W_{0}+\frac{\left(b_{1}+b_{2}\right)\left(W_{2}-W_{1}\right)-2 \int_{-\infty}^{x} d x^{\prime} \frac{\partial}{\partial y}\left(W_{2}\left(x^{\prime}, y\right)-W_{1}\left(x^{\prime}, y\right)\right)}{b_{1}-b_{2}+W_{1}-W_{2}} .
$$

For $\partial W / \partial y=0$ this formula is reduced to the well-known superposition formula 
for the Korteweg-de Vries family of the equations (see e.g. Ref. [20]).

\section{Conclusion}

1. In addition to problem (1.1), there exists an other generalisation of the GelfandDickey spectral problem to two dimensions, namely the problem

$$
\frac{\partial^{N} \chi}{\partial \cdot x^{N}}+V_{N-2}(x, y, t) \frac{\partial^{N-2} \chi}{\partial \cdot x^{N-2}}+\cdots+V_{0}(x, y, t) \chi-\frac{\partial^{N} \chi}{\partial y^{N}}=0 .
$$

Spectral problem (7.1) can also be obtained as a result of the $\tilde{Z}_{N}$ reduction of the general matrix problem $\partial \Psi / \partial x+A \partial \Psi / \partial y+P(x, y, t) \Psi=0$ (see [14]).

For the two-dimensional problem (7.1), one can obtain all the relations analogous to those given in the Sect.2. But the recursion operators of the type $\hat{\Lambda}_{n}$ and $\check{\Lambda}_{n}$ (with the properties (3.24) and (3.26)) do not exist for problem (7.1). Therefore an essential modification of our constructions is needed for the applicability of the AKNS-technique to problem (7.1).

2. Let us note also that in the present paper we consider the direct scattering problem for (1.1) by treating the variable $x$ as a time type variable, i.e. the scattering matrix $S$ connects the asymptotics of the solutions $\chi$ of problem (1.1) at $x$-infinites (at $x=+\infty$ and $x=-\infty$ ). In [18] in the case $N=2$ (i.e. for $\partial \chi / \partial y+\partial^{2} \chi / \partial x^{2}+$ $V(x, y, t) \chi=0)$ the standard version of the scattering problem for the nonstationary Schrödinger equation was used in which a scattering matrix connects the solutions at $y$-infinites, i.e. at $y=+\infty$ and $y=-\infty$. The interrelation between these two approaches will be considered elsewhere.

\section{References}

1. Zakharov, V. E., Manakov, S. V., Novikov, S. P., Pitaevski, L. P.: Soliton Theory. Method of the Inverse Problem, Moscow: Nauka 1980 (in Russian)

2. Solitons: In: Topics in Current Physics, Vol. 17, Bullough, R., Caudrey, P. (eds.) Berlin, Heidelberg, New York: Springer 1980

3. Ablowitz, M. J., Kaup, D. J., Newell, A. C., Segur, H. : Stud. Appl. Math. 53, 249 (1974)

4. Miodek, I.: IST-solvable nonlinear evolution equations and existence-An extension of Lax's method. J. Math. Phys. 19, 19 (1978)

5. Newell, A. C.: The general structure of integrable evolution equations. Proc. R. Soc. (London) A365, 283 (1979)

6. Kulish, P. P.: Notes of LOMI scientific seminars, 96, 105 (1980) (in Russian)

7. Konopelchenko, B. G.: The linear spectral problem of arbitrary order: The general form of the integrable equations and their Bäcklund transformations. Phys. Lett. 75A. 447 (1980); On the structure of integrable evolution equations. Phys. Lett. 79A, 39 (1980); On the structure of the commutative $Z_{2}$ graded algebra valued integrable equations. Phys. Lett. 95B, 83 (1980); Transformation properties of the integrable evolution equations. Phys. Lett. 100B, 254 (1981)

8. Konopelchenko, B. G.: On the structure of equations integrable by the arbitrary-order linear spectral problem, J. Phys. A: Math. Gen. 14, 1237 (1981)

9. Konopelchenko, B. G.: On the general structure of integrable equations and $Z_{\mathrm{N}^{-}}$-type reductions. Phys. Lett., 108B, 26 (1982); preprint Institute of Nuclear Physics 81-99 (1981)

10. Gerdjikov, V. S., Kulish, P. P.: The generating operator for the $n \times n$ linear system. Physica 3D, 549 (1981)

11. Gerdjikov, V. S., Ivanov, M. I., Kulish, P. P.: Teor. Mat. Fyz. 44, 342 (1980) (in Russian) 
12. Konopelchenko, B. G.: The polynomial spectral problem of arbitrary order: A general form of the integrable equations and Bäcklund transformations. J. Phys. A.: Math. Gen. 14, 3125 (1981)

13. Dubrovsky, V. G. Konopelchenko, B. G.: Preprint Institute of Nuclear Physics 82-9 (1982)

14. Konopelchenko, B. G.: The two-dimensional matrix spectral problem: General structure of the integrable equations and their Bäcklund transformations. Phys. Lett. 86A, 346 (1981)

On the general structure of nonlinear evolution equations integrable by the two-dimensional matrix spectral problem. Commun. Math. Phys. 87, 105-125 (1982)

15. Zakharov, V. E., Shabat, A. B.: Funct. Anal. 8, No. 3, 43 (1974) (in Russian)

16. Zakharov, V. E.: The Inverse Scattering Method. In: Solitons, Ref. [2], p. 243

17. Dryuma, V. S.: Analytic solution of the two-dimensional Korteweg-de Vries (KdV) equation. JETP Lett. 19, 387 (1974)

18. Manakov, S. V.: The Inverse Scattering Transform for the Time-Dependent Schrödinger Equation and Kadomtsev-Petviashvili Equation, In: Proc. of Soviet-American Symposium on Soliton Theory, September 1979, Kiev; Physica 3D, 420 (1981)

19. Chudnovsky, D. V.: In: Lecture Notes in Physics. Vol. 120, pp. 103 Berlin, Heidelberg, New York: Springer 1980

20. Bäcklund Transformations. The Inverse Scattering Method, Solitons and Their Applications, R. Miura, (ed.) In: Lecture Notes in Mathematics, Vol. 515, New York: Springer 1976

21. Chen. H.-H. : A Bäcklund Transformation in Two Dimensions. J. Math. Phys. 16, 2382 (1975)

Communicated by Ya. G. Sinai

Received March 15, 1982 
